
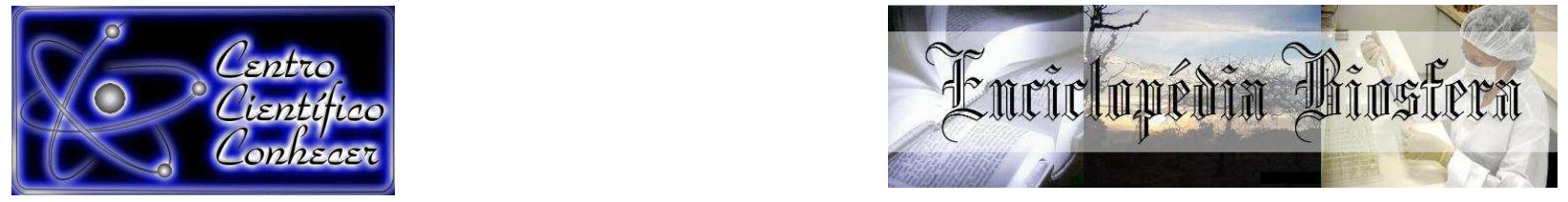

\title{
ENTOMOFAUNA ASSOCIADA AO CADÁVER DE SUÍNO SUS SCROFA LINNAEUS (SUIDAE) NO MUNICÍPIO DE CURUÇÁ, PARÁ, BRASIL
}

José Augusto Melo Ribeiro ${ }^{1}$, Ana Lúcia Nunes Gutjahr², Carlos Elias de Souza Braga $^{3}$, Antônio Carlos Santos Melo ${ }^{4}$

1 Graduado em Ciências Naturais, Universidade do Estado do Pará. Belém, Pará, Brasil.

2 Professora Doutora, Departamento de Ciências Naturais, Universidade do Estado do Pará(melcam@uol.com.br). Belém, Pará, Brasil.

3 Professor Doutor, Departamento de Ciências Naturais, Universidade do Estado do Pará. Belém, Pará, Brasil.

4 Graduado em Ciências Naturais, Universidade do Estado do Pará. Belém, Pará,

Recebido em: 02/10/2017 - Aprovado em: 21/11/2017 - Publicado em: 05/12/2017 DOI: 10.18677/EnciBio 2017B82

A Entomologia Forense associa insetos e outros artrópodes a procedimentos legais, principalmente relacionados à decomposição cadavérica. O presente trabalho fornece informações da entomofauna associada à decomposição de suíno em ambiente natural na cidade de Curuçá, Pará. Foram realizados dois experimentos no período mais chuvoso e menos chuvoso, característicos da região amazônica. Foi, usado como modelo experimental dois cadáveres de porco doméstico de aproximadamente $15 \mathrm{~kg}$, sob uma gaiola de metal, cuja finalidade era proteger a carcaça suína de animais carnívoros de grande porte. Os insetos associados ao processo de decomposição foram coletados com rede entomológica e com armadilhas de pitffal e identificados em nível de família. Os resultados mostraram que os insetos pertencentes às ordens Diptera tiveram maior abundância, seguidos de Hymenoptera e Coleoptera, ratificando a importância forense dessas ordens em investigações relacionadas à decomposição cadavérica. Fatores abióticos como temperatura e precipitação também influenciaram na entomofauna associada à decomposição cadavérica estudada. Ressalta-se que o conhecimento das espécies envolvidas nos processos de decomposição nas diversas regiões geográficas do Brasil é um passo importantíssimo para uma efetiva utilização dos insetos como ferramentas para a solução de crimes.

PALAVRAS-CHAVE: Decomposição cadavérica; Entomologia Forense; Insetos; Amazônia.

\section{ASSOCIATED ENTOMOFAUNA WITH A PIG CADAVER SUS SCROFA (SUIDAE) IN THE MUNICIPALITY OF CURUÇÁ, PARÁ, BRAZIL}

\begin{abstract}
The Forensic Entomology associates insects and other arthropods to legal procedures, mainly related to cadaveric decomposition. This paper provides information to the associated entomofauna with pig decomposition in natural environment in the town of Curuçá, Para. Two one experiments were carried out in
\end{abstract}


the rainy season and the other with little rainfall, characteristic for the Amazon region. For the experimental model two domestic pig carcasses of approximately $15 \mathrm{~kg}$ were used within a metal cage, whose purpose was to protect the pig carcass of large carnivores. Insects associated with the process of decomposition were collected with entomological net and pitffal traps and identified at family level. The results showed that the insects belonging to Diptera were most abundant, followed by Hymenoptera and Coleoptera, confirming the forensic importance of these orders in investigations related to the cadaveric decomposition. Abiotic factors such as temperature and precipitation also influenced the associated entomofauna with cadaveric decomposition. It is emphasized that the knowledge of the species involved in the processes of decomposition in the various geographic regions of Brazil is a major step for effective use of insects as a tool for solving criminal matters.

KEYWORDS: Cadaveric decomposition; Forensic entomology; Insects; Amazon.

\section{INTRODUÇÃO}

Estudos relacionados à entomologia forense utilizam em suas análises, insetos pertencentes, principalmente, as ordens Diptera, Coleoptera e Hymenoptera ocorrentes na área onde o cadáver se encontra (OLIVEIRA-COSTA, 2011). A ordem Diptera é uma das maiores e mais diversas dentre os insetos e a mais importante na área forense (OLIVEIRA-COSTA et al., 2013). A ordem Coleoptera é considerada a segunda ordem mais frequente e abundante na colonização de cadáveres em decomposição, possuindo muitos representantes necrófagos que são fundamentais na determinação do intervalo pós-morte (IPM), especialmente nos estágios mais secos do processo de decomposição (OLIVEIRA-COSTA, 2011). Os Hymenoptera são frequentes em pesquisas forenses, sendo a família Formicidae a mais representativa (PANIGALLI; SOLIGO, 2013), correspondendo a mais de $85 \%$ da ordem associada à área forense, podendo retardar o processo de decomposição quando atua como predadora, de outros insetos necrófagos.

$\mathrm{Na}$ entomologia forense, os insetos são importantes aliados, no entanto é preciso ter mais conhecimento sobre as ordens, famílias e espécies que se associam a cadáveres em decomposição nos mais diversos ambientes e biomas. Portanto, estudos relacionados a essa área são importantes, pois fornecem informações que podem auxiliar em investigações criminais, mas, para isso, faz-se necessário pelo menos um estudo local experimental com modelo animal para que se obtenham dados relacionados à entomofauna local específica e o tempo de decomposição em determinado ambiente, que podem variar dependendo das condições climáticas (AMENDT et al., 2007).

O presente trabalho teve como objetivo determinar a entomofauna associada aos processos de decomposição cadavérica do suíno Sus scrofa Linnaeus em ambiente natural no município de Curuçá, Estado do Pará, durante dois períodos distintos do ano, um mais chuvoso e outro menos chuvoso, característicos da região Amazônica.

\section{Área de Estudo}

\section{MATERIAL E MÉTODOS}

Os experimentos foram realizados no Município de Curuçá, (0045'38,8" S / $47^{\circ} 50^{\prime} 56,5^{\prime}$ W), localizado no Nordeste do Estado do Pará, Brasil, a uma altitude de 29m. (Figura 1) em um terreno com vegetação secundária (capoeira baixa), em ambiente rural, distante $5 \mathrm{~km}$ do centro da cidade sede do município, com acesso pela rodovia PA-136. 
O clima da região onde se localiza a cidade de Curuçá pode ser caracterizado como tropical úmido. A umidade relativa do ar média é de $80 \%$, com a temperatura variando ao longo do ano de $26,8^{\circ} \mathrm{C}$ a $28,0^{\circ} \mathrm{C}$. As maiores precipitações ocorrem em fevereiro, março e abril e as menores nos meses de setembro, outubro e novembro. Portanto, caracterizando dois períodos sazonais distintos na região (mais chuvoso e menos chuvoso), nos quais foram realizados os experimentos. Segundo classificação climática de Köppen-Geiger, o clima de Curuçá é do tipo Am, com a temperatura média anual de $26,2{ }^{\circ} \mathrm{C}$ e pluviosidade média anual de $2327 \mathrm{~mm}$ (Climate-Data.org, 2015).

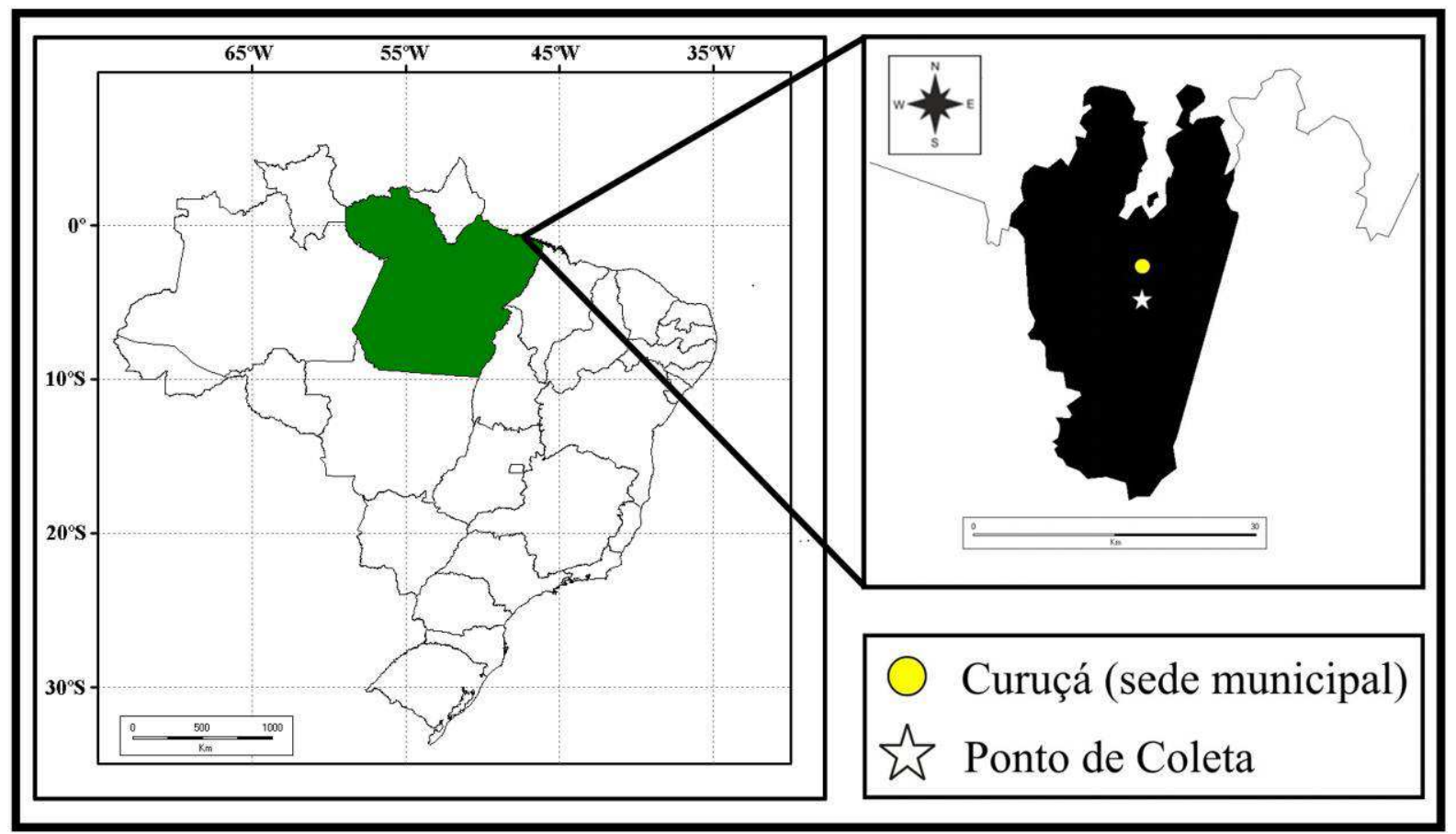

FIGURA 1. Localização do Município de Curuçá e do ponto de experimentação na área de estudo.

\section{Experimentação e coleta de dados}

O estudo foi realizado em 2013, durante os meses de abril e maio, característicos do período mais chuvoso, e setembro e outubro, os quais são característicos do período menos chuvoso. Foram utilizados dois porcos domésticos (Sus scrofa) de aproximadamente $15 \mathrm{~kg}$ como modelos experimentais, um para cada período do ano (mais chuvoso e menos chuvoso). A justificativa para a escolha do modelo é devido à semelhança desses animais com os humanos quanto à anatomia interna, plasma e hábito alimentar (onivoria), tamanho da cavidade torácica e quantidade de pelos.

A carcaça do animal foi mantida em contato com o solo sob uma gaiola de metal medindo $1,0 \times 1,0 \times 1,0 \mathrm{~m}$, revestida com tela de 3,0 $\times 3,0 \mathrm{~cm}$, confeccionada para permitir a entrada de insetos e impedir o acesso de vertebrados de grande porte, existentes na área de estudo. Os insetos alados que visitaram a carcaça durante o processo de decomposição foram coletados manualmente com o auxílio de uma rede entomológica. Para a coleta dos insetos rasteiros foram utilizadas seis armadilhas de solo tipo pitfall contendo água e sabão líquido, dispostas em volta da gaiola com carcaça em forma de círculo a um raio aproximado de 1,5 metros da mesma (Figura 2). 
As coletas dos insetos (imaturos e adultos) durante os experimentos se deram em intervalos de 3 horas nas primeiras 24 horas, após isso, diariamente, nos horários das $9 \mathrm{~h}$ às $12 \mathrm{~h}$ até o início da fase de esqueletização, e posteriormente de dois em dois dias até completar 30 dias de experimento.

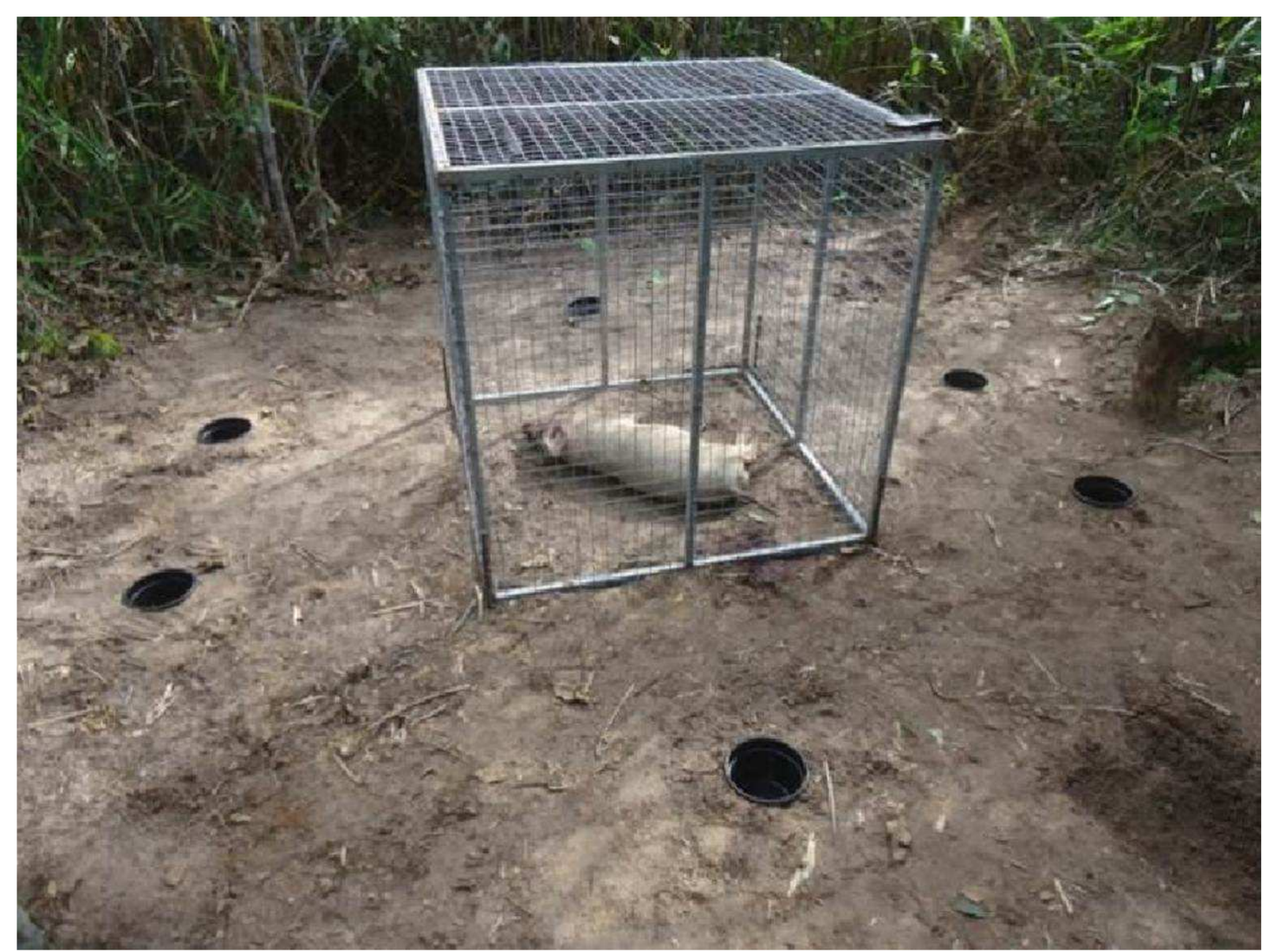

FIGURA 2. Aspecto da gaiola de experimentação e das armadilhas de pitfall's na área de estudo em Curuçá, Pará, 2013.

As informações meteorológicas diárias referentes aos períodos do desenvolvimento dos experimentos, quanto a temperaturas máxima e mínima e índice pluviométrico foram obtidos diariamente no portal eletrônico Tempo Agora (www.tempoagora.com.br). Em complemento a essas informações, foram coletados os dados referentes à precipitação a partir de uma estação local do Serviço Geológico do Brasil (CPRM), localizada as proximidades do experimento, para verificar se os dados locais correspondem ou se assemelham aos boletins fornecidos pelo site, permitindo definir a possibilidade de seu uso confiável em situações futuras não experimentais (Tabela 1). 
TABELA 1. Listagem dos dados abióticos (temperatura e pluviosidade) nos períodos: mais chuvoso (abril e maio) e menos chuvoso (setembro e outubro) em Curuçá, Pará, 2013.

\begin{tabular}{|c|c|c|c|}
\hline Data & $\begin{array}{c}\text { Temp } \\
\text { Min. }\end{array}$ & $\begin{array}{l}\text { Temp } \\
\text { Máx. }\end{array}$ & Precipitação \\
\hline $12 / 04 / 2013$ & $25^{\circ} \mathrm{C}$ & $31^{\circ} \mathrm{C}$ & $4,7 \mathrm{~mm}$ \\
\hline $13 / 04 / 2013$ & $27^{\circ} \mathrm{C}$ & $29^{\circ} \mathrm{C}$ & $2,5 \mathrm{~mm}$ \\
\hline $14 / 04 / 2013$ & $27^{\circ} \mathrm{C}$ & $33^{\circ} \mathrm{C}$ & $0,1 \mathrm{~mm}$ \\
\hline $15 / 04 / 2013$ & $26^{\circ} \mathrm{C}$ & $33^{\circ} \mathrm{C}$ & $0,2 \mathrm{~mm}$ \\
\hline $16 / 04 / 2013$ & $26^{\circ} \mathrm{C}$ & $29^{\circ} \mathrm{C}$ & $2 \mathrm{~mm}$ \\
\hline $17 / 04 / 2013$ & $26^{\circ} \mathrm{C}$ & $28^{\circ} \mathrm{C}$ & $27,2 \mathrm{~mm}$ \\
\hline $18 / 04 / 2013$ & $27^{\circ} \mathrm{C}$ & $28^{\circ} \mathrm{C}$ & $8,3 \mathrm{~mm}$ \\
\hline $19 / 04 / 2013$ & $25^{\circ} \mathrm{C}$ & $30^{\circ} \mathrm{C}$ & $53 \mathrm{~mm}$ \\
\hline 20/04/2013 & $26^{\circ} \mathrm{C}$ & $33^{\circ} \mathrm{C}$ & $4,7 \mathrm{~mm}$ \\
\hline 21/04/2013 & $25^{\circ} \mathrm{C}$ & $30^{\circ} \mathrm{C}$ & $56,8 \mathrm{~mm}$ \\
\hline $22 / 04 / 2013$ & $26^{\circ} \mathrm{C}$ & $28^{\circ} \mathrm{C}$ & $0,2 \mathrm{~mm}$ \\
\hline 23/04/2013 & $27^{\circ} \mathrm{C}$ & $33^{\circ} \mathrm{C}$ & $0,9 \mathrm{~mm}$ \\
\hline $24 / 04 / 2013$ & $27^{\circ} \mathrm{C}$ & $30^{\circ} \mathrm{C}$ & $15,6 \mathrm{~mm}$ \\
\hline $25 / 04 / 2013$ & $27^{\circ} \mathrm{C}$ & $33^{\circ} \mathrm{C}$ & $1,7 \mathrm{~mm}$ \\
\hline $26 / 04 / 2013$ & $25^{\circ} \mathrm{C}$ & $27^{\circ} \mathrm{C}$ & $3,4 \mathrm{~mm}$ \\
\hline 27/04/2013 & $24^{\circ} \mathrm{C}$ & $27^{\circ} \mathrm{C}$ & $71,1 \mathrm{~mm}$ \\
\hline $28 / 04 / 2013$ & $25^{\circ} \mathrm{C}$ & $28^{\circ} \mathrm{C}$ & $25,4 \mathrm{~mm}$ \\
\hline 4/2013 & $25^{\circ} \mathrm{C}$ & $27^{\circ} \mathrm{C}$ & $3,6 \mathrm{~mm}$ \\
\hline $30 / 04 / 2013$ & $25^{\circ} \mathrm{C}$ & $33^{\circ} \mathrm{C}$ & $103,4 \mathrm{~mm}$ \\
\hline 01/05/2013 & $25^{\circ} \mathrm{C}$ & $32^{\circ} \mathrm{C}$ & $21,1 \mathrm{~mm}$ \\
\hline 02/05/2013 & $25^{\circ} \mathrm{C}$ & $33^{\circ} \mathrm{C}$ & $24,8 \mathrm{~mm}$ \\
\hline 03/05/2013 & $27^{\circ} \mathrm{C}$ & $33^{\circ} \mathrm{C}$ & $0,1 \mathrm{~mm}$ \\
\hline 04/05/2013 & $25^{\circ} \mathrm{C}$ & $29^{\circ} \mathrm{C}$ & $18,9 \mathrm{~mm}$ \\
\hline 05/05/2013 & $26^{\circ} \mathrm{C}$ & $32^{\circ} \mathrm{C}$ & $13,2 \mathrm{~mm}$ \\
\hline 06/05/2013 & $25^{\circ} \mathrm{C}$ & $31^{\circ} \mathrm{C}$ & $21,1 \mathrm{~mm}$ \\
\hline $07 / 05 / 2013$ & $27^{\circ} \mathrm{C}$ & $31^{\circ} \mathrm{C}$ & $2,9 \mathrm{~mm}$ \\
\hline 08/05/2013 & $26^{\circ} \mathrm{C}$ & $28^{\circ} \mathrm{C}$ & $3,8 \mathrm{~mm}$ \\
\hline $09 / 05 / 2013$ & $27^{\circ} \mathrm{C}$ & $28^{\circ} \mathrm{C}$ & $10,2 \mathrm{~mm}$ \\
\hline $10 / 05 / 2013$ & $26^{\circ} \mathrm{C}$ & $30^{\circ} \mathrm{C}$ & $6,6 \mathrm{~mm}$ \\
\hline $11 / 05 / 2013$ & $26^{\circ} \mathrm{C}$ & $32^{\circ} \mathrm{C}$ & $14,7 \mathrm{~mm}$ \\
\hline
\end{tabular}

\begin{tabular}{|c|c|c|c|}
\hline Data & $\begin{array}{c}\text { Temp } \\
\text { Min. }\end{array}$ & $\begin{array}{l}\text { Temp } \\
\text { Máx. }\end{array}$ & Precipitação \\
\hline $20 / 09 / 2013$ & $28^{\circ} \mathrm{C}$ & $32^{\circ} \mathrm{C}$ & $0 \mathrm{~mm}$ \\
\hline 21/09/2013 & $27^{\circ} \mathrm{C}$ & $33^{\circ} \mathrm{C}$ & $0 \mathrm{~mm}$ \\
\hline $22 / 09 / 2013$ & $27^{\circ} \mathrm{C}$ & $33^{\circ} \mathrm{C}$ & $0 \mathrm{~mm}$ \\
\hline 23/09/2013 & $24^{\circ} \mathrm{C}$ & $32^{\circ} \mathrm{C}$ & $0,1 \mathrm{~mm}$ \\
\hline 24/09/2013 & $27^{\circ} \mathrm{C}$ & $32^{\circ} \mathrm{C}$ & $0 \mathrm{~mm}$ \\
\hline $25 / 09 / 2013$ & $27^{\circ} \mathrm{C}$ & $29^{\circ} \mathrm{C}$ & $0,1 \mathrm{~mm}$ \\
\hline 26/09/2013 & $27^{\circ} \mathrm{C}$ & $33^{\circ} \mathrm{C}$ & $0 \mathrm{~mm}$ \\
\hline 27/09/2013 & $27^{\circ} \mathrm{C}$ & $32^{\circ} \mathrm{C}$ & $0 \mathrm{~mm}$ \\
\hline 28/09/2013 & $26^{\circ} \mathrm{C}$ & $29^{\circ} \mathrm{C}$ & $0 \mathrm{~mm}$ \\
\hline 29/09/2013 & $25^{\circ} \mathrm{C}$ & $30^{\circ} \mathrm{C}$ & $0 \mathrm{~mm}$ \\
\hline $30 / 09 / 2013$ & $24^{\circ} \mathrm{C}$ & $32^{\circ} \mathrm{C}$ & $0,3 \mathrm{~mm}$ \\
\hline $01 / 10 / 2013$ & $26^{\circ} \mathrm{C}$ & $33^{\circ} \mathrm{C}$ & $0 \mathrm{~mm}$ \\
\hline 02/10/2013 & $26^{\circ} \mathrm{C}$ & $32^{\circ} \mathrm{C}$ & $0 \mathrm{~mm}$ \\
\hline $03 / 10 / 2013$ & $24^{\circ} \mathrm{C}$ & $32^{\circ} \mathrm{C}$ & $0 \mathrm{~mm}$ \\
\hline 04/10/2013 & $25^{\circ} \mathrm{C}$ & $32^{\circ} \mathrm{C}$ & $0 \mathrm{~mm}$ \\
\hline $05 / 10 / 2013$ & $27^{\circ} \mathrm{C}$ & $33^{\circ} \mathrm{C}$ & $0 \mathrm{~mm}$ \\
\hline 06/10/2013 & $27^{\circ} \mathrm{C}$ & $32^{\circ} \mathrm{C}$ & $0 \mathrm{~mm}$ \\
\hline $07 / 10 / 2013$ & $28^{\circ} \mathrm{C}$ & $32^{\circ} \mathrm{C}$ & $0 \mathrm{~mm}$ \\
\hline 08/10/2013 & $24^{\circ} \mathrm{C}$ & $33^{\circ} \mathrm{C}$ & $0 \mathrm{~mm}$ \\
\hline $09 / 10 / 2013$ & $25^{\circ} \mathrm{C}$ & $32^{\circ} \mathrm{C}$ & $0 \mathrm{~mm}$ \\
\hline $10 / 10 / 2013$ & $25^{\circ} \mathrm{C}$ & $33^{\circ} \mathrm{C}$ & $0 \mathrm{~mm}$ \\
\hline $11 / 10 / 2013$ & $25^{\circ} \mathrm{C}$ & $32^{\circ} \mathrm{C}$ & $0 \mathrm{~mm}$ \\
\hline $12 / 10 / 2013$ & $24^{\circ} \mathrm{C}$ & $32^{\circ} \mathrm{C}$ & $0 \mathrm{~mm}$ \\
\hline $13 / 10 / 2013$ & $25^{\circ} \mathrm{C}$ & $33^{\circ} \mathrm{C}$ & $2,1 \mathrm{~mm}$ \\
\hline $14 / 10 / 2013$ & $25^{\circ} \mathrm{C}$ & $33^{\circ} \mathrm{C}$ & $0 \mathrm{~mm}$ \\
\hline $15 / 10 / 2013$ & $26^{\circ} \mathrm{C}$ & $32^{\circ} \mathrm{C}$ & $0 \mathrm{~mm}$ \\
\hline $16 / 10 / 2013$ & $25^{\circ} \mathrm{C}$ & $33^{\circ} \mathrm{C}$ & $0 \mathrm{~mm}$ \\
\hline $17 / 10 / 2013$ & $25^{\circ} \mathrm{C}$ & $31^{\circ} \mathrm{C}$ & $0 \mathrm{~mm}$ \\
\hline $18 / 10 / 2013$ & $26^{\circ} \mathrm{C}$ & $32^{\circ} \mathrm{C}$ & $0 \mathrm{~mm}$ \\
\hline $19 / 10 / 2013$ & $26^{\circ} \mathrm{C}$ & $32^{\circ} \mathrm{C}$ & $0 \mathrm{~mm}$ \\
\hline & \multicolumn{2}{|c|}{$\begin{array}{c}\text { Média } \\
\text { temperatura } \\
29^{\circ} \mathrm{C}\end{array}$} & $\begin{array}{c}\text { Média } \\
\text { precipitação } \\
0,086 \mathrm{~mm}\end{array}$ \\
\hline
\end{tabular}

A identificação dos insetos atraídos pela carcaça foi realizada em nível de ordem e família, segundo Triplehorn e Jonnson (2011), Rafael et al. (2012). Todo o material coletado foi depositado na Coleção Zoológica Didático-Científica da Universidade do Estado do Pará (UEPA).

\section{RESULTADOS E DISCUSSÃO}

Durante o período de realização dos experimentos de decomposição cadavérica, foram coletados 1329 insetos, pertencentes a nove ordens e 39 famílias. O maior número de famílias foi observado para as ordens Coleoptera e Diptera, sendo 11 e 10 famílias respectivamente (Tabela 2). Do total de indivíduos capturados, 402 espécimes (30,25\%) foram coletados no período mais chuvoso e 
$927(69,75 \%)$ no período menos chuvoso. Dessa forma, percebe-se que a maioria dos insetos coletados possui maior relação com o período mais seco, assim como observado por Rosa et al. (2009), quando no experimento, realizado em UberlândiaMG, observaram maior diversidade e equitabilidade na distribuição das espécies no período seco.

TABELA 2. Ordens, famílias e número de espécimes de Insecta coletados em Carcaça de Sus scofra durante os períodos mais chuvoso e menos chuvoso em Curuçá, Pará, 2013.

\begin{tabular}{|c|c|c|c|c|c|c|}
\hline \multicolumn{2}{|c|}{ Entomofauna coletada } & \multicolumn{2}{|c|}{ Mais chuvoso } & \multicolumn{2}{|c|}{ Menos chuvoso } & \multirow[t]{2}{*}{ Total } \\
\hline ORDEM & FAMÍLIA & CARC 1 & PIT 1 & CARC 2 & PIT 2 & \\
\hline \multirow{10}{*}{ Diptera } & Calliphoridae & 104 & 15 & 173 & 37 & 329 \\
\hline & Sarcophagidae & 2 & 0 & 9 & 3 & 14 \\
\hline & Muscidae & 13 & 0 & 18 & 2 & 33 \\
\hline & Phoridae & 1 & 0 & 34 & 5 & 40 \\
\hline & Empididae & 1 & 0 & 0 & 2 & 3 \\
\hline & Sepsidae & 1 & 0 & 3 & 0 & 4 \\
\hline & Ephidridae & 4 & 0 & 0 & 0 & 4 \\
\hline & Tephritidae & 0 & 1 & 0 & 0 & 1 \\
\hline & Stratiomyidae & 0 & 0 & 5 & 2 & 7 \\
\hline & Dilichopodidae & 0 & 0 & 0 & 2 & 2 \\
\hline \multirow{11}{*}{ Coleoptera } & Scarabaeidae & 6 & 23 & 15 & 90 & 134 \\
\hline & Nitidulidae & 8 & 6 & 31 & 141 & 186 \\
\hline & Staphylinidae & 4 & 1 & 0 & 10 & 15 \\
\hline & Tenebrionidae & 4 & 22 & 3 & 0 & 29 \\
\hline & Carabidae & 2 & 0 & 0 & 0 & 2 \\
\hline & Rysodidae & 1 & 0 & 0 & 0 & 1 \\
\hline & Chysomelidae & 2 & 0 & 0 & 0 & 2 \\
\hline & Silphidae & 0 & 0 & 3 & 0 & 3 \\
\hline & Lucanidae & 0 & 0 & 1 & 0 & 1 \\
\hline & Lampiridae & 0 & 1 & 0 & 0 & 1 \\
\hline & Bruchidae & 0 & 1 & 0 & 0 & 1 \\
\hline \multirow{3}{*}{ Hymenoptera } & Formicidae & 34 & 76 & 107 & 164 & 381 \\
\hline & Aphidae & 18 & 0 & 14 & 1 & 33 \\
\hline & Vespidae & 0 & 1 & 1 & 3 & 5 \\
\hline \multirow[t]{5}{*}{ Blattodea } & Blaberidae & 0 & 2 & 0 & 7 & 9 \\
\hline & Blattelidae & 0 & 0 & 1 & 12 & 13 \\
\hline & Blattidae & 1 & 0 & 0 & 4 & 5 \\
\hline & Gryllidae & 0 & 10 & 0 & 4 & 14 \\
\hline & Acrididae & 0 & 0 & 0 & 1 & 1 \\
\hline \multirow{3}{*}{ Orthoptera } & Gryllacdidae & 0 & 0 & 0 & 1 & 1 \\
\hline & Romaleidae & 0 & 1 & 0 & 0 & 1 \\
\hline & Tettigoniidae & 0 & 1 & 0 & 0 & 1 \\
\hline Isoptera & Kalotermitidae & 30 & 0 & 9 & 3 & 42 \\
\hline \multirow[t]{2}{*}{ Dermaptera } & Labiduridae & 0 & 0 & 0 & 1 & 1 \\
\hline & Forficulidae & 0 & 0 & 0 & 2 & 2 \\
\hline \multirow[t]{2}{*}{ Lepidoptera } & Microlepdoptera & 0 & 0 & 0 & 1 & 1 \\
\hline & Fulgoridae & 0 & 0 & 0 & 1 & 1 \\
\hline \multirow[t]{2}{*}{ Hemiptera } & Reduviidae & 1 & 3 & 1 & 0 & 5 \\
\hline & Coreidae & 0 & 1 & 0 & 0 & 1 \\
\hline TOTAL & & 237 & 165 & 428 & 499 & 1329 \\
\hline
\end{tabular}


Entre os insetos coletados, os dípteros com 32,88\%, himenópteros com $31,75 \%$ e coleópteros com $27,99 \%$ se destacaram como as três ordens mais abundantes, estando entre os grupos mais influentes no processo de decomposição nos experimentos realizados no município de Curuçá. As outras ordens de insetos capturadas sumarizam pouco mais de $7 \%$ do total coletado.

Entre as famílias de dípteros identificados, os mais relevantes, com maior percentual de exemplares foram Calliphoridae (75,28\%), Phoridae $(9,15 \%)$, Muscidae $(7,55 \%)$ e Sarcophagidae $(3,2 \%)$. Entre os coleópteros identificados, as famílias Nitidulidae $(50 \%)$, Scarabaeidae $(36,02 \%)$, Tenebrionidae $(7,79 \%)$ e Staphylinidae $(4,03 \%)$ foram as mais abundantes. Os himenópteros foram representados por três famílias distintas, Formicidae $(90,28 \%)$, Apidae $(7,81 \%)$ e Vespidae com menos de $2 \%$ do total coletado.

A diversidade de famílias de insetos que visitaram as carcaças foi semelhante entre os experimentos dos períodos mais chuvoso e menos chuvoso, sendo evidente o domínio da ordem Diptera, seguido de Hymenoptera e Coleotera, nos dois períodos.

Os resultados observados estão de acordo com as informações Pacini et al. (2015), quando afirmam que entre os artrópodes necrófagos a ordem Diptera é a mais frequente, seguida pela ordem Coleoptera, as quais participam ativamente do processo de decomposição, alimentando-se da matéria orgânica que serve tanto de fonte proteica, como sítio de cópula e estímulo à oviposição. Divergindo em partes dessas informações, quando a segunda ordem mais frequente nos dois períodos estudados foi Hymenoptera, sendo representada por mais de $90 \%$ de formigas. Em muitos estudos, as formigas se apresentam em grande abundância, entre os himenópteros coletados em carcaças, destacando a importância desse grupo em pesquisas forenses (FIGUEIRA; SOUTO, 2015).

Em pesquisas forenses é fundamental se levar em conta às condições climáticas diferenciadas durante os períodos de experimentação, as quais para este estudo realizado em Curuçá, os parâmetros médios foram: temperatura de $27^{\circ} \mathrm{e}$ precipitação de $17,4 \mathrm{~mm}$ no período mais chuvoso e $28,5^{\circ}$ de temperatura e $0,086 \mathrm{~mm}$ de precipitação no período menos chuvoso, visto que, tais fatores tendem a influenciar diretamente na sucessão ecológica nas carcaças. Pois foi notória a diferenciação da ocorrência das famílias durante as diferentes fases de decomposição do suíno, nos períodos mais chuvoso e menos chuvoso (Quadro 1).

QUADRO 1. Ocorrência de insetos associados às diferentes fases de decomposição e suíno na cidade de Curuçá, Pará, durante os períodos mais chuvoso (C) e menos chuvoso (MC) em 2013.

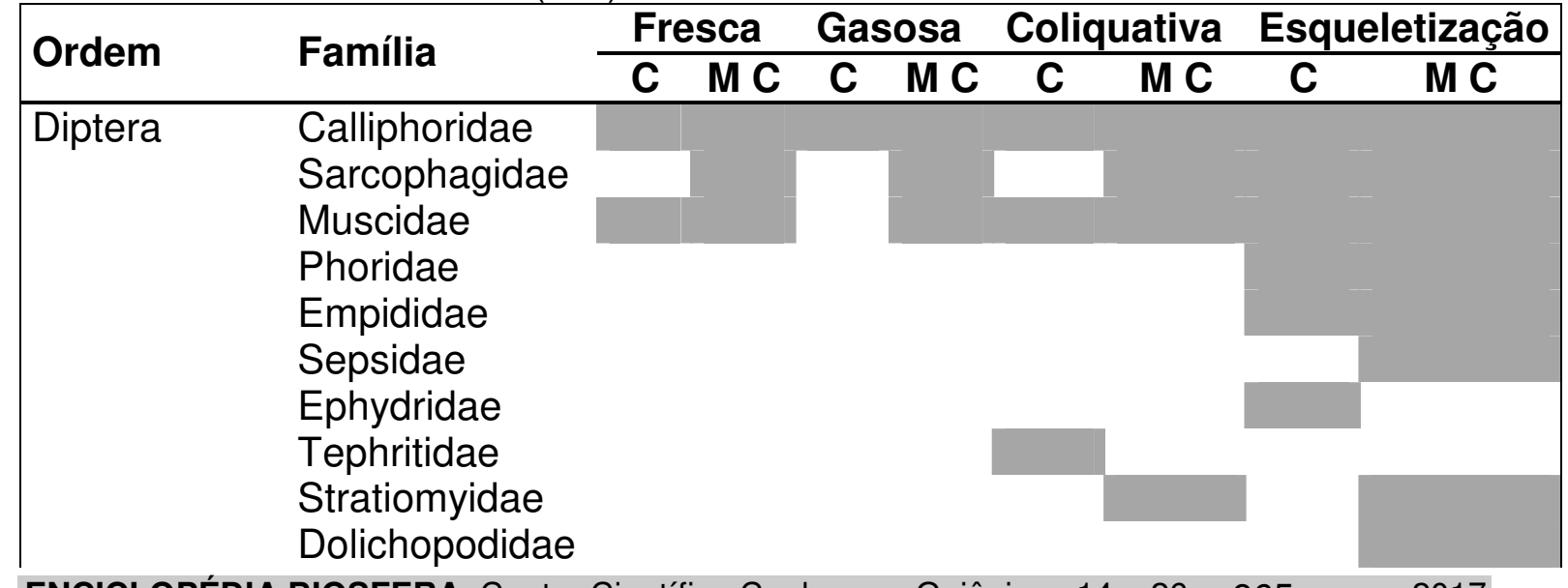




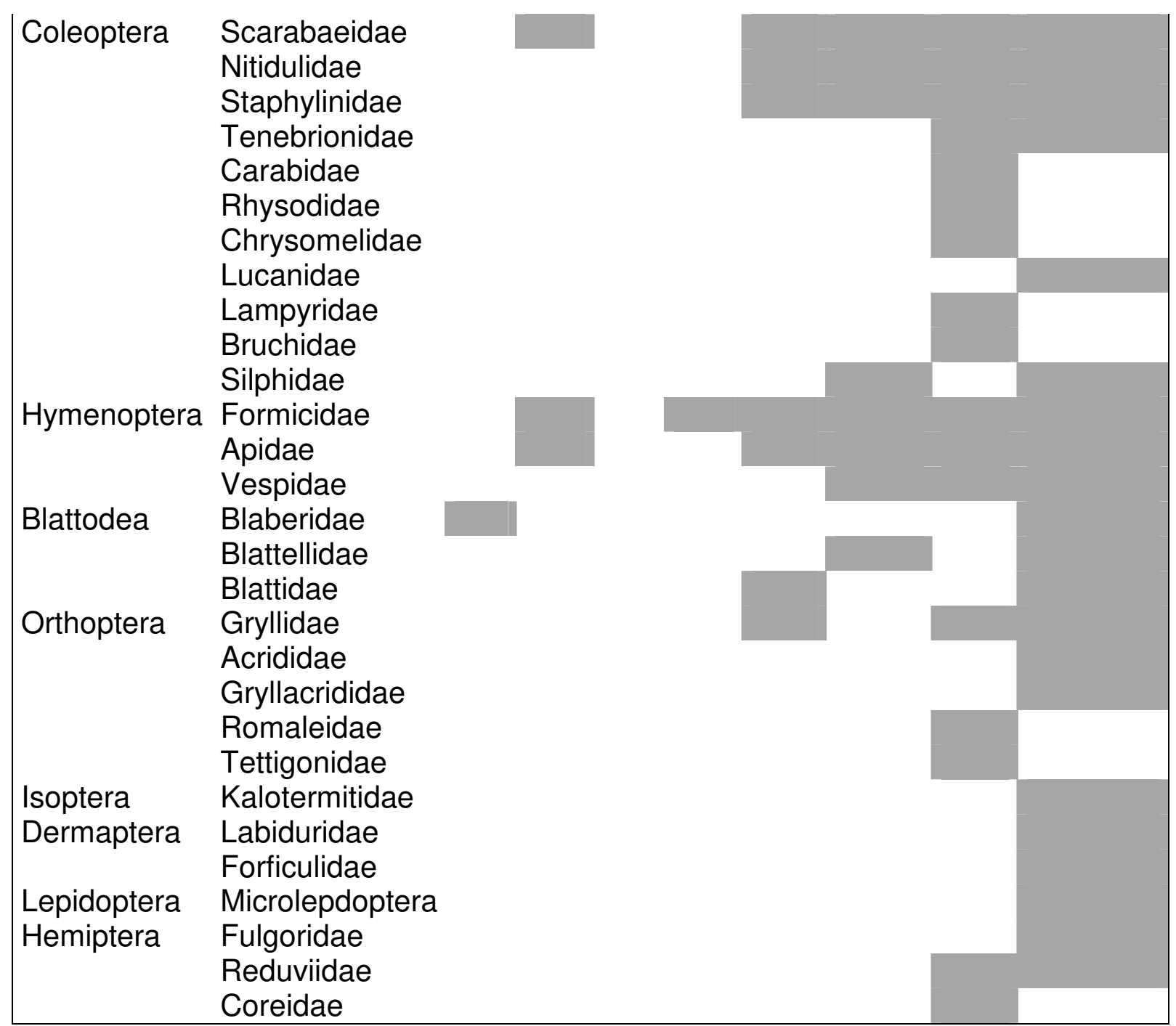

No período mais chuvoso, logo após a morte do animal, houve imediata aproximação de dípteros junto à carcaça, constatando-se que as primeiras famílias a frequentá-la foram Calliphoridae e Muscidae. No período menos chuvoso, também houve imediata aproximação de dípteros, porém das famílias Calliphoridae, Sarcophagidae e Muscidae. De acordo com os dados de Oliveira-Costa (2011), os adultos dessa ordem são tipicamente sinantrópicos e extremamente ágeis, razão pela qual são os primeiro insetos a chegar ao cadáver e algumas espécies ao atingem a carcaça depositam seus ovos, em pouco tempo após a morte do animal.

A ordem Diptera esteve presente em todas as fases de decomposição de ambos os experimentos, sendo a família Calliphoridae a única a frequentar todas as fases nos dois períodos. Esses dados estão de acordo com Oliveira-Costa et al. (2013) quando observaram que califorídeos não apresentam preferência quanto à estação do ano, visto que ocorrem em todos os estágios de decomposição, sendo essa família considerada um importante indicador forense.

Neste estudo, os dípteros coletados na carcaça e nas armadilhas de pitfall durante o período mais chuvoso ( $n=142$ indivíduos) e no menos chuvoso ( $n=295$ indivíduos), observou-se que a Família mais frequente em ambos os períodos foi Calliphoridae com 119 indivíduos no mais chuvoso e 210 indivíduos no menos chuvoso (Figuras 3 e 4). Quanto a isso, Gredilha e Melo (2008) em estudos com Calliphoridae no Rio de Janeiro admitem que a pluviosidade seja o fator abiótico que 
influencia as populações desse muscoide, o que pode reforçar os resultados obtidos neste estudo. Ressalta-se, ainda, que Gonçalves et al. (2011), afirmam que os fatores climáticos podem ocasionar mudanças no comportamento populacional de algumas espécies de Calliphoridae.

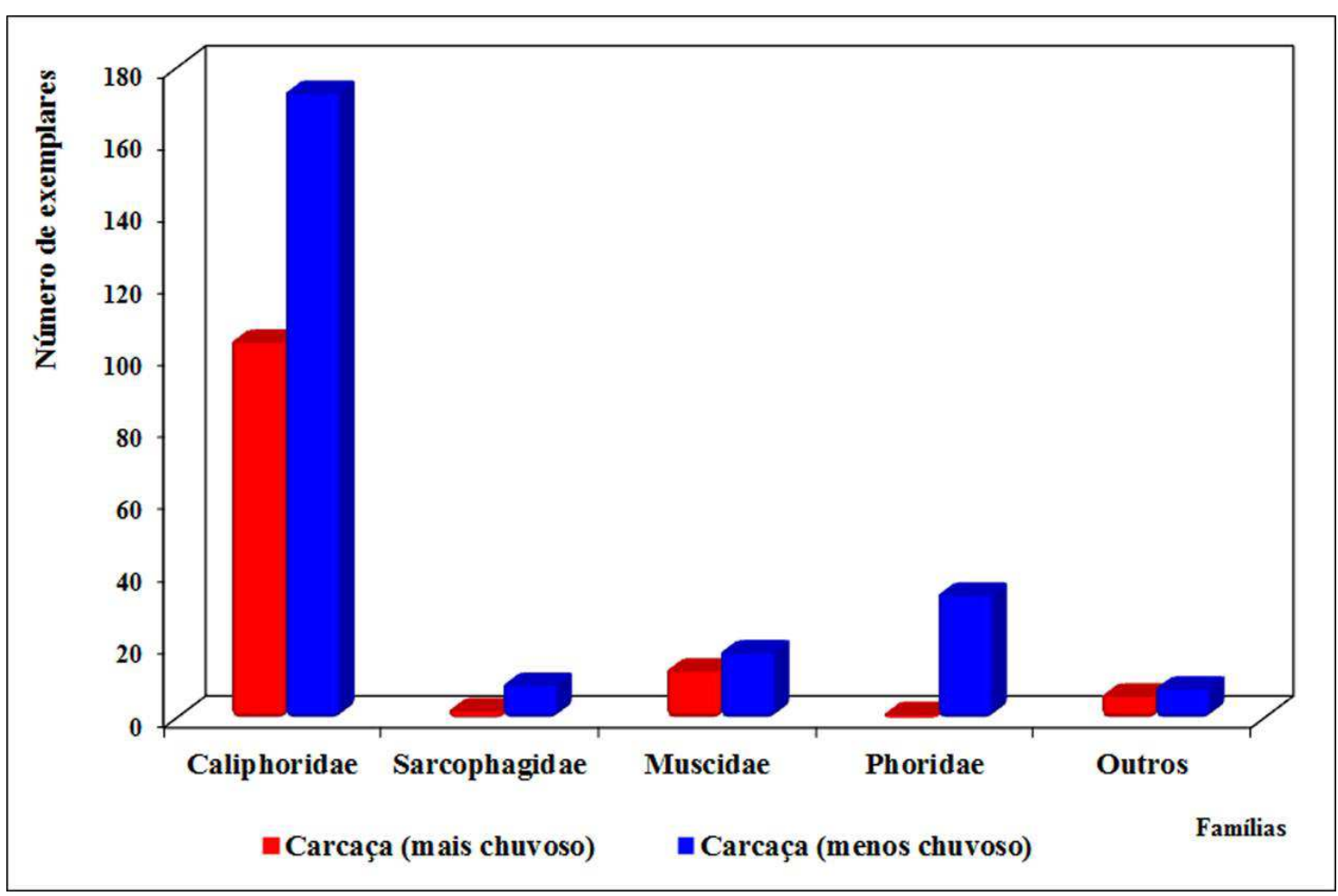

FIGURA 3. Principais Famílias de Diptera coletados nas carcaças de suíno durante os períodos mais chuvoso e menos chuvoso em Curuçá, Pará, em 2013.

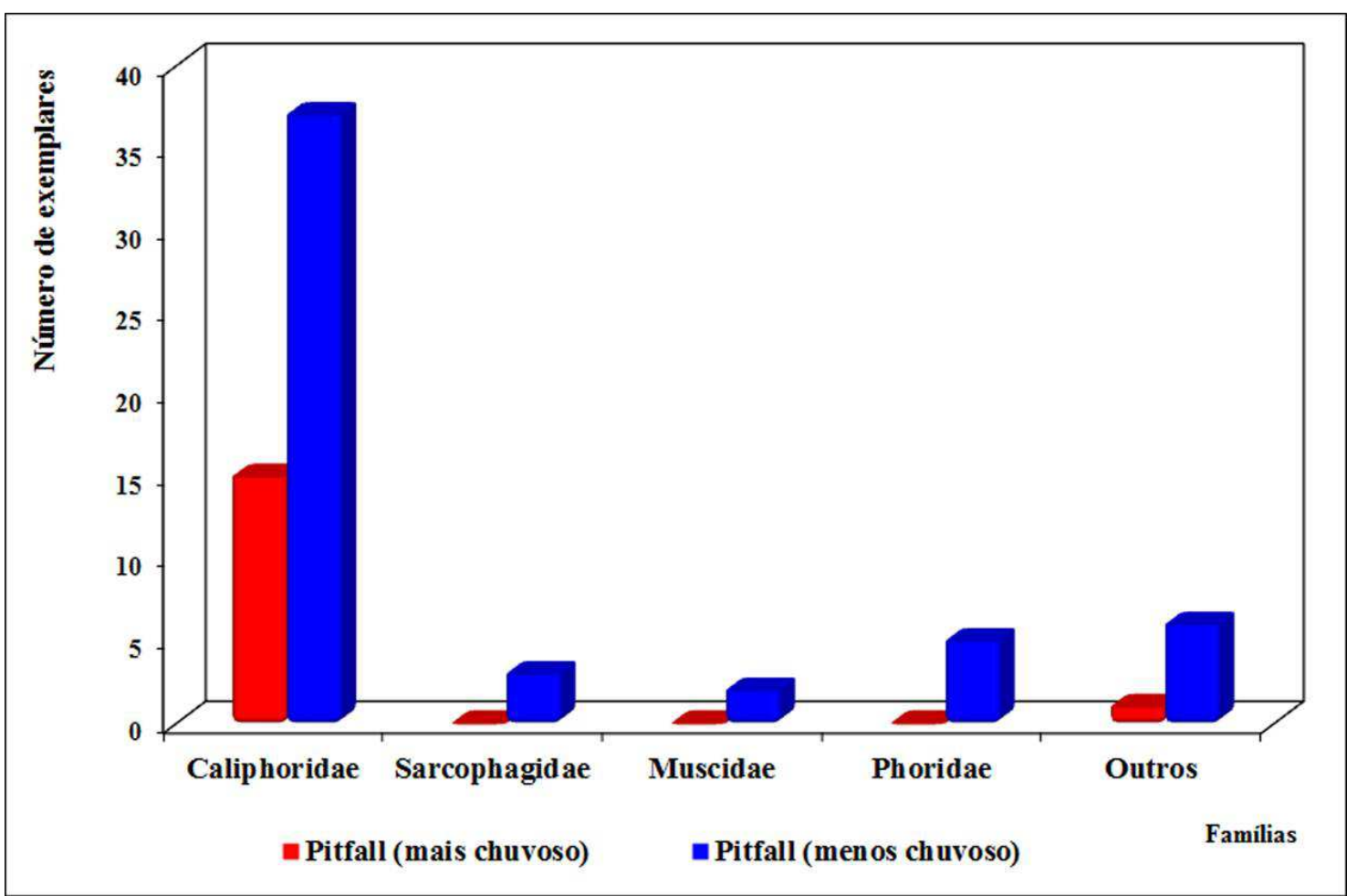

FIGURA 4. Principais Famílias de Diptera coletados nos pitfall's durante os períodos chuvoso e menos chuvoso em Curuçá, Pará, em 2013. 
Durante os experimentos, observou-se uma grande quantidade de emergência de Calliphoridae, sendo um número superior às demais famílias. Ururahy-Rodrigues (2013), no Amazonas, e Silva (2013), no extremo Sul do Brasil, também coletaram uma grande quantidade de califorídeos associados às carcaças em decomposição. Portanto essa família pode ser associada ao processo de decomposição em diversas regiões geográficas do Brasil, indicando também, que pode ser considerada dominante em relação às outras famílias de muscoides.

Os himenópteros estiveram presentes no período mais chuvoso a partir da fase coliquativa, entretanto no período menos chuvoso estiveram em todas as fases de decomposição. Formicidae foi a família mais abundante nos dois períodos. Este resultado é corroborado pelo estudo realizado por Amendt et al. (2007) que constataram a presença de Formicidae em todos os estágios de decomposição, reforçando a importância desse grupo em pesquisas forenses. No período mais chuvoso foram capturados 129 himenópteros de três famílias distintas sendo Formicidae a mais abundante com mais de $85 \%$ dos indivíduos. Já no período menos chuvoso foram capturados 293 indivíduos, sendo mais de $90 \%$ de Formicidae (Figuras 5 e 6 ).

Neste estudo a abundância de Formicidae nos dois períodos (mais chuvoso e menos chuvoso) reforça a importância forense desses insetos também em Curuçá, assim como no trabalho de Cruz e Vasconselos (2006), onde Hymenoptera foi a mais frequente, sendo Formicidae mais abundante, ressaltando ainda mais a relevância desse grupo na decomposição de cadáveres. Algumas famílias de Hymenoptera, principalmente Formicidae, estão diretamente relacionadas ao processo de decomposição, pois coloniza a carcaça, onde nessas cadeias alimentares, estas podem ser necrófagas, predadoras ou onívoras, (OLIVEIRACOSTA 2011), destacando a grande e significativa importância forense desses insetos.

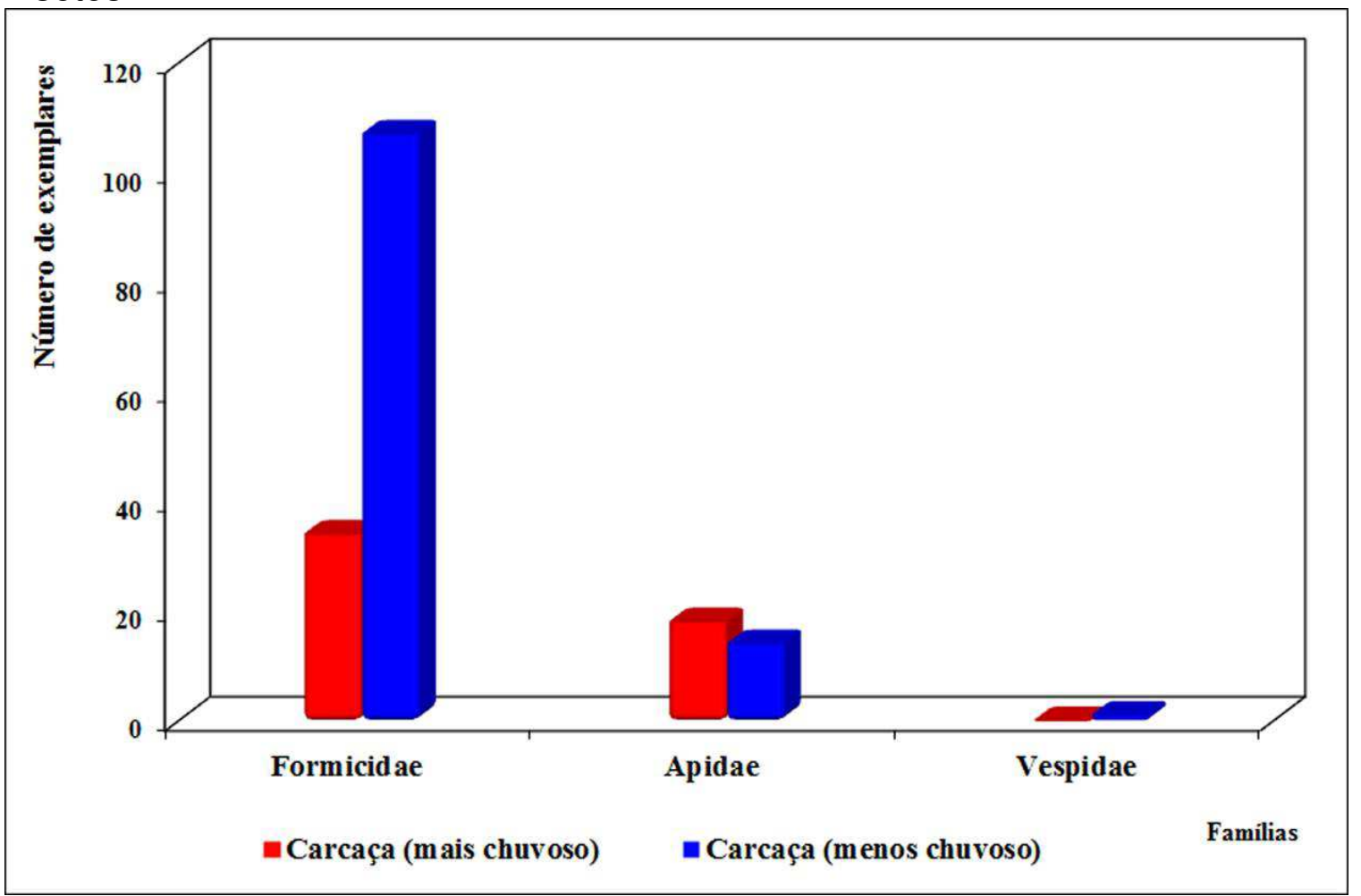

FIGURA 5. Famílias de Hymenoptera coletados nas carcaças de suíno durante os períodos mais chuvoso e menos chuvoso em Curuçá, Pará, em 2013. 


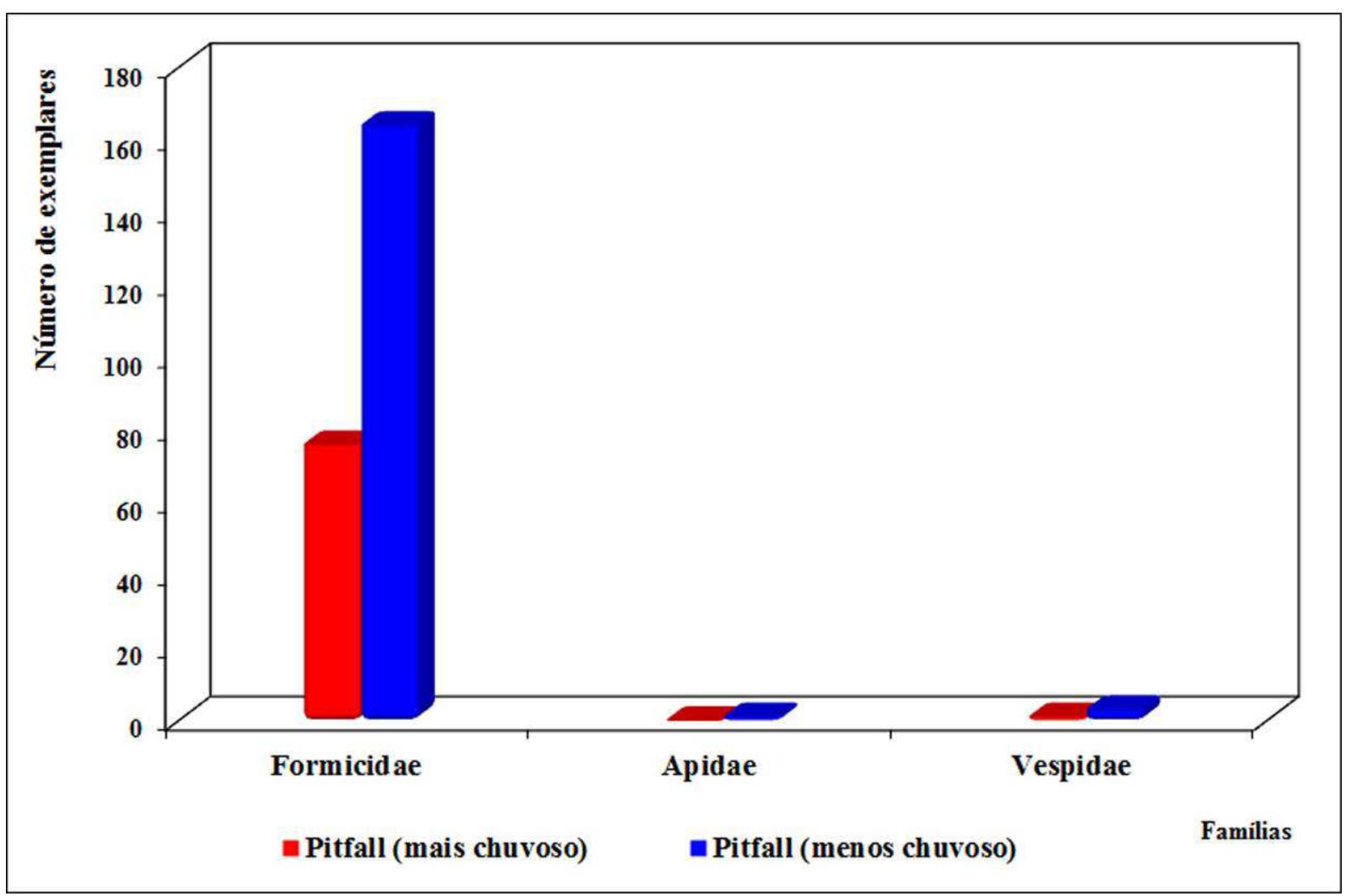

FIGURA 6. Famílias de Hymenoptera coletados nos pitfall's durante os períodos mais chuvoso e menos chuvoso em Curuçá, Pará, em 2013.

Os grupos de Coleoptera mostraram preferência pelas fases finais do processo de decomposição, principalmente a esqueletização. Segundo OliveiraCosta (2011), os coleópteros colonizam carcaças em decomposição, principalmente nos estágios secos do processo, quando os cadáveres já foram abandonados pelos dípteros, o que corrobora o observado nos experimentos de Curuçá. No período mais chuvoso coletou-se um total de 81 indivíduos de Coleoptera pertencentes a nove famílias, sendo as mais abundantes Scarabaeidae com 29 indivíduos, Tenebrionidae com 26 e Nitidulidae com 14. No período menos chuvoso, foram coletados 291 indivíduos, destacando-se como mais abundantes Nitidulidae com 172 amostras, Scarabaeidae com 105 e Staphylinidae com 10 (Figuras 7 e 8). 


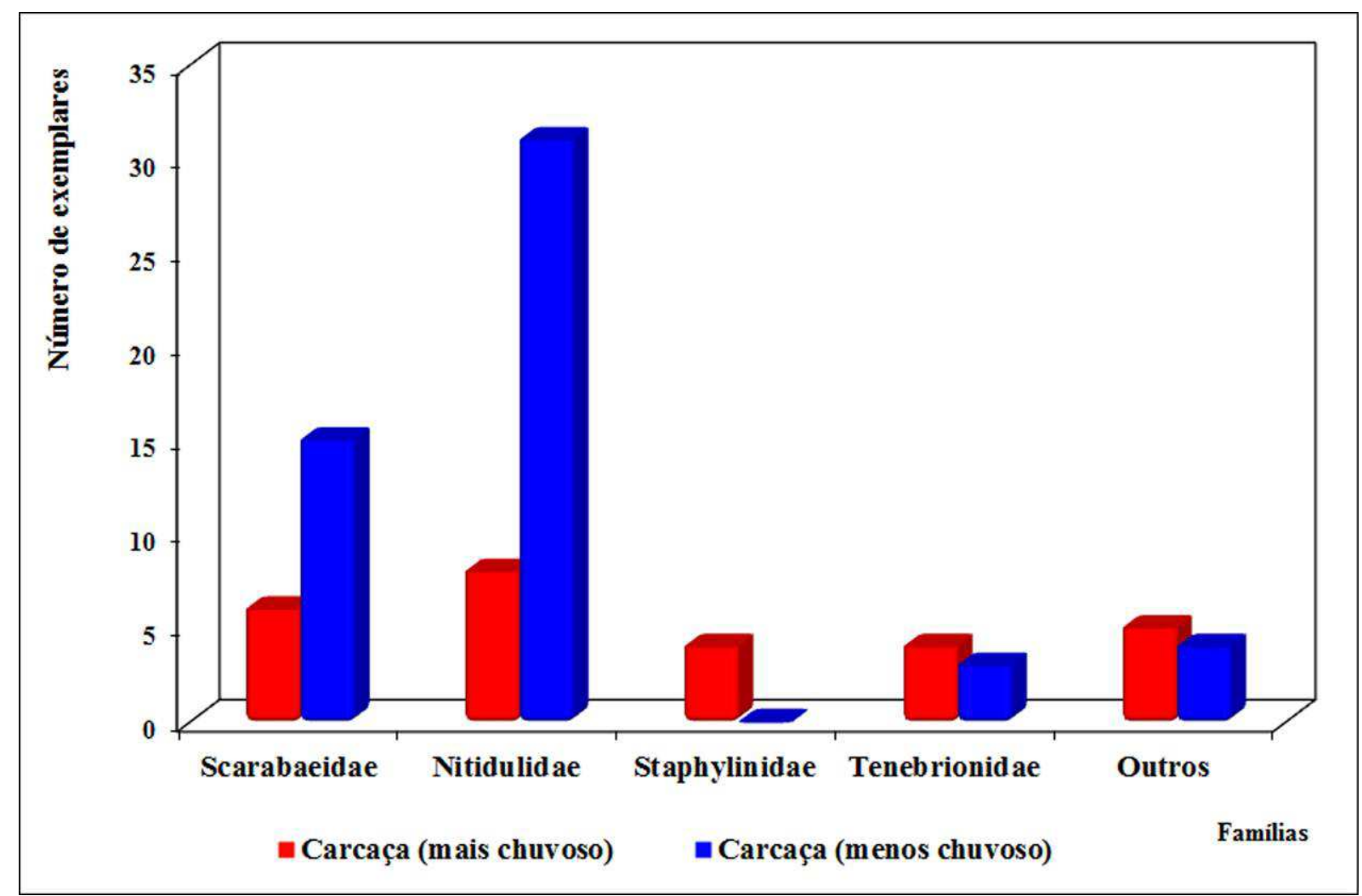

FIGURA 7. Principais Famílias de Coleoptera coletados nas carcaças de suíno durante os períodos mais chuvoso e menos chuvoso em Curuçá, Pará, em 2013.

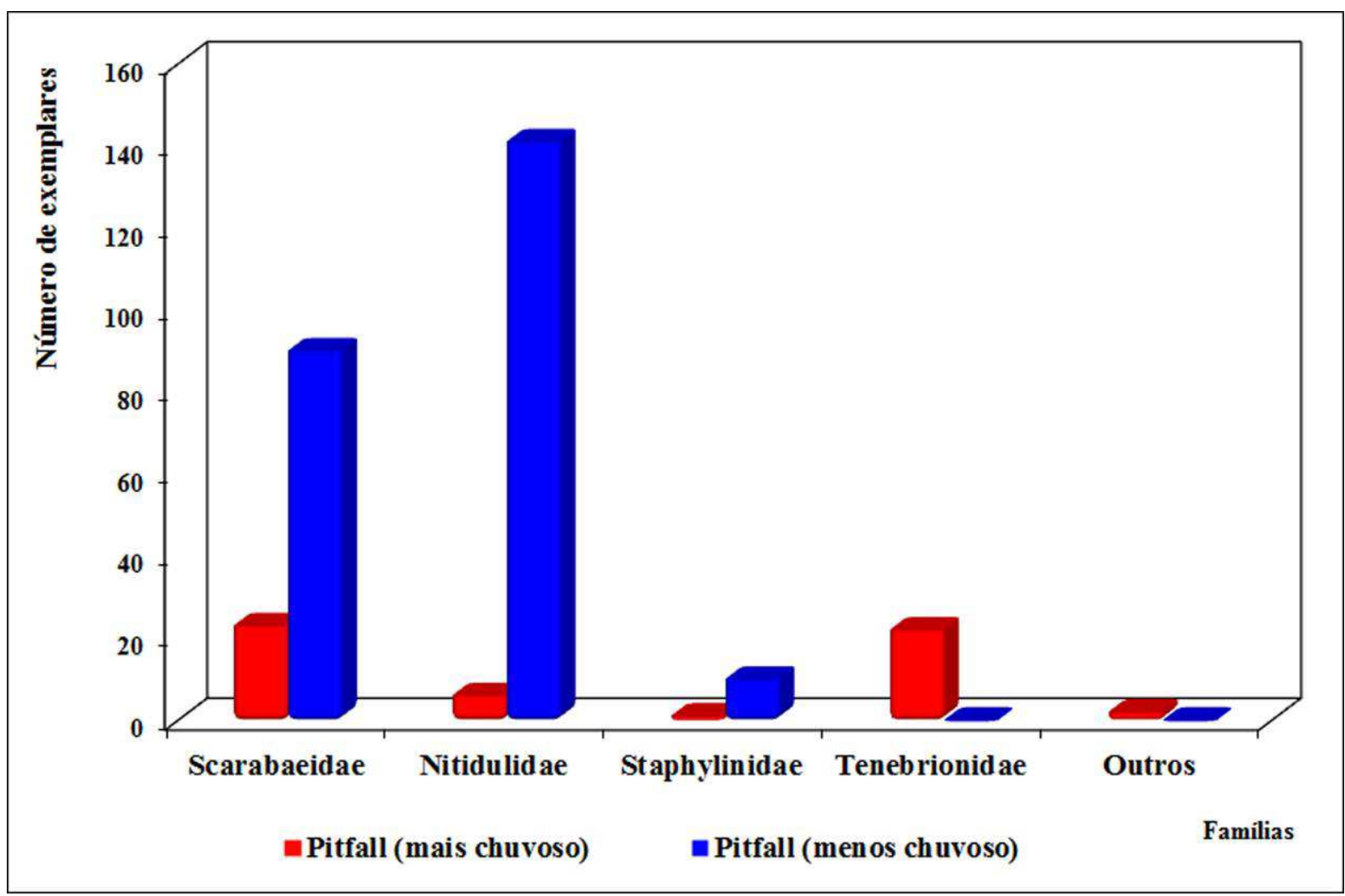

FIGURA 8. Principais Famílias de Coleoptera coletados nos pitfall's durante os períodos mais chuvoso e menos chuvoso em Curuçá, Pará, em 2013. 


\section{CONCLUSÃo}

Os resultados obtidos nos experimentos ratificaram a importância forense das ordens Diptera, Coleoptera e Hymenoptera, em investigações relacionadas à decomposição cadavérica. Cabe ressaltar, que o conhecimento das espécies envolvidas nos processos de decomposição nas diversas regiões geográficas do Brasil é um passo importantíssimo para uma efetiva utilização dos insetos como ferramenta para a solução de crimes. Considerando que, o Brasil possui grandes dimensões geográficas, e com isso, clima e fauna decompositora diferenciada para cada região, os dados obtidos nesse estudo são importantes para determinar a entomofauna decompositora na região do município de Curuçá.

Além disso, pode-se dizer que a ocorrência de determinadas famílias de insetos nas diferentes fases de decomposição pode variar de acordo com as condições climáticas, sendo assim, a ocorrência de insetos no processo de decomposição cadavérica de suíno em Curuçá, está diretamente influenciada por fatores abióticos como a temperatura e precipitação.

Sabe-se que uma grande diversidade de insetos está relacionada à decomposição de cadáveres. Nesse sentido é importante que se tenha conhecimento do ciclo de vida dos insetos, hábitos e sucessões ecológicas na cena do crime. No entanto, para que se monte um banco de dados que reúna dados confiáveis sobre a fauna decompositora, nas diversas regiões geográficas do Brasil, que possa ser utilizado como referência em investigações de âmbito legal relacionadas a crimes, principalmente na estimativa de intervalo pós-morte (IPM), estudos mais aprofundados em diferentes regiões ainda se fazem necessários.

\section{AGRADECIMENTOS}

Os autores agradecem ao proprietário do sítio por ter permitido que os experimentos deste estudo tenham se realizado em sua propriedade.

\section{REFERÊNCIAS}

AMENDT, J.; CAMPOBASSO, C. P.; GAUDRY, E.; REITER, C.; LEBLANC, H. N.; HALL, M.J.R. Best practice in forensic entomology - standards and guidelines. International journal of legal Medicine, v. 121, p. 90-104, 2007. Disponível em: <http://dx.doi.org/10.1007/s00414-006-0086-x> doi: 10.1007/s00414-006-0086-x.

CLIMATE-DATA.ORG. 2015. Clima: Curuçá. Disponível em: <http://pt.climatedata.org/location/29790>. Acesso: 02 out. 2017.

CRUZ, T. M.; VASCONCELOS, S. D. Entomofauna de solo associada à decomposição de carcaça de suíno em um fragmento de mata atlântica de Pernambuco, Brasil. Biociências, v. 14, p. 193-201, 2006.

FIGUEIRA, S. S.; SOUTO, R. N. P. Entomologia Forense: histórico e contextualização no Estado do Amapá. Biota Amazônia, v. 5, n. 1, p. 123-127, 2015. Disponível em: <http://dx.doi.org/10.18561/21795746/biotaamazonia.v5n1p123-127> 5746/biotaamazonia.v5n1p123-127.

doi:

$10.18561 / 2179$

GONÇALVES, L.; DIAS, A.; ESPINDOLA, C. B.; ALMEIDA, F. S. Inventário de Calliphoridae (Diptera) em manguezal e fragmento de Mata Atlântica na região de 
Barra de Guaratiba, Rio de Janeiro, Brasil. Revista Brasileira de Biociências, v. 9, p. 50-55, 2011.

GREDILHA, R.; MELLO, R. P. Sinantropia de dípteros muscóides (Diptera, Calliphoridae) no Município de Paracambi-RJ. Revista Brasileira de Zoociências, v. 10 , p. $217-221,2008$.

OLIVEIRA-COSTA, J., Entomologia Forense - Quando os insetos são vestígios. Campinas, Milennium, 520 p., 2011.

OLIVEIRA-COSTA, J.; OLIVEIRA, R. G.; BASTOS, C. S. Diptera Calliphoridae de importância forense no município do Rio de Janeiro. Revista Eletrônica Novo Enfoque, v. 16, n. 16, p. 41-52, 2013.

PACINI, D. B.; SANTOS, C. R. M.; TEIXEIRA, C. R.; QUEIROZ, P. R. M. Uso dos diptera na análise entomotoxicológica e na estimativa do intervalo pós-morte (IPM). Universitas: Ciências da Saúde, v. 13, p. 29-39, 2015. Disponível em: <http://dx.doi.org/10.5102/ucs.v13i1.2846> doi: 10.5102/ucs.v13i1.2846.

PANIGALLI, G.; SOLIGO, K. T. Diversidade de Insecta (Arthropoda) associada à carcaça de Sus scrofa I. Em um fragmento de Mata Atlântica de Xanxerê, Santa Catarina. Unoesc \& Ciência - ACBS, v. 4, n. 1, p. 15-26, 2013.

RAFAEL, J. A.; MELO, G. A. R.; de CARVALHO, C. J. B.; CASARI S. A.; CONSTANTINO, R. Insetos do Brasil: Diversidade e Taxonomia. Holos Editora, Ribeirão Preto, 810 p., 2012.

ROSA, T. A.; BABATA, M. L. Y.; SOUZA, C. M.; SOUSA; D.; MELLO-PATIU, C. A.; MENDES, J. Dípteros de Interesse Forense em Dois Perfis de Vegetação de Cerrado em Uberlândia, MG. Neotropical Entomology, v. 38, n. 6, p. 859-866, 2009.

SILVA, T. N.; QUEIROZ, P. R. M. Características da família Sarcophagidae aplicadas à análise forense. Universidade Católica de Goiás, v. 1, p. 1-18, 2013. Disponível em: <http://www.cpgls.pucgoias.edu.br/6mostra/artigos/SAUDE/THARA\%20NUNES\%20SILVA\% 20E\%20PAULO\%20ROBERTO\%20QUEIROZ.pdf>. Acesso em: 02 out. 2017.

TEMPO AGORA. Disponível em: <http://www.tempoagora.com.br/> Acesso em: 02 out. 2017.

TRIPLEHORN, C. A.; JOHNSON, N. F. Estudo dos insetos - tradução da 7"a edição de borror and delong's introduction to the study of insects. São Paulo, Cengage Learning, 809p., 2011.

URURAHY-RODRIGUES, A.; PUJOL-LUZ, J. R.; RAFAEL, J. A. Temporal distribution of blowflies of forensic importance (Diptera: Calliphoridae), in man-size domestic pigs carcasses, in the Forest Reserve Adolpho Ducke, Manaus, Amazonas, Brazil. EntomoBrasilis, v. 6: p. 09-22, 2013. Disponível em: <http://dx.doi.org/doi:10.12741/ebrasilis.v6i1.242>. doi:10.12741/ebrasilis.v6i1.242. 